\title{
Analysis of Text Translation Strategies in Medical English
}

\author{
Chen Yifang \\ Xiamen Huaxia University, China,361000
}

Keywords: Medical English; text translation; translation strategy

Abstract: The sources of medical English texts are wide, the forms are diverse and the content is complex, which leads to many problems in the translation process of medical English texts. This paper firstly makes a reasonable classification of medical English texts and then applies appropriate translation strategies for different types of medical English selection, so that the translation achieves the effect and purpose of "faithfulness, expressiveness, elegance".

Medical English generally refers to the research, teaching, application and services of medical personnel and related medical departments in the field of clinical medicine, including the terminology used in medical practice. In the development of medical career in China, the translation of medical English has always played a very important role in boosting. Since joining the WTO, China's international exchanges in the medical field have become increasingly frequent. The status of Chinese medicine in the international arena continues to rise and the speed of updating medical knowledge is also accelerating. Therefore, the demand for professional medical text translation is increasing. This also requires medical English translators to be systematically trained and trained to meet the growing needs ${ }^{[1]}$.

\section{Classification of medical English text}

\subsection{Names of places and names}

In the course of the development of Western medicine, a group of researchers and medical experts who have made outstanding contributions to the medical cause have emerged. To commemorate these great medical scientists, their research results are often named after the names of the main researchers. Or, named after the place where the scientific research was born, for example, Addison's anemia, Edison Anemia (Malignant Anemia).

\subsection{Animal names}

There are three main manifestations of this type of medical English: (1) a virus or disease is highly susceptible to infection and can be transmitted to humans, such as bird flu avian influenza; (2) similar to a human population Animal malformations, such as chicken breast; (3) Name the medical special phenomenon with the unique unique characteristics of a particular animal, such as frog breathing frog breathing (sphincter breathing) and seagull murmur Sexual noise, etc. 


\subsection{Plant name class}

This type of medical English also has three main forms of expression: (1) the characteristics of a disease caused by a certain plant's unique shape, color, etc., such as banana sign; (2) Name the disease caused by the name of a plant, such as betel cancer betel nut cancer (wild leaf cheek cancer); (3) name the drug directly using the name of the plant, such as Pollen Pini pine pollen ${ }^{[2]}$.

\subsection{Color class}

Different words expressing color can represent different things in medical English. In addition, abnormalities in physiological phenomena can also be distinguished by words that express color, such as red blood cell, black tongue and black tongue.

\subsection{Shape class}

In medical English, words or roots representing shapes are often used to express the state of related things, such as triangular muscle deltoid muscle; cricoid cartilage ring cartilage, balloon cell balloon-like cells and basket cell basket cells.

\subsection{Quantifier class}

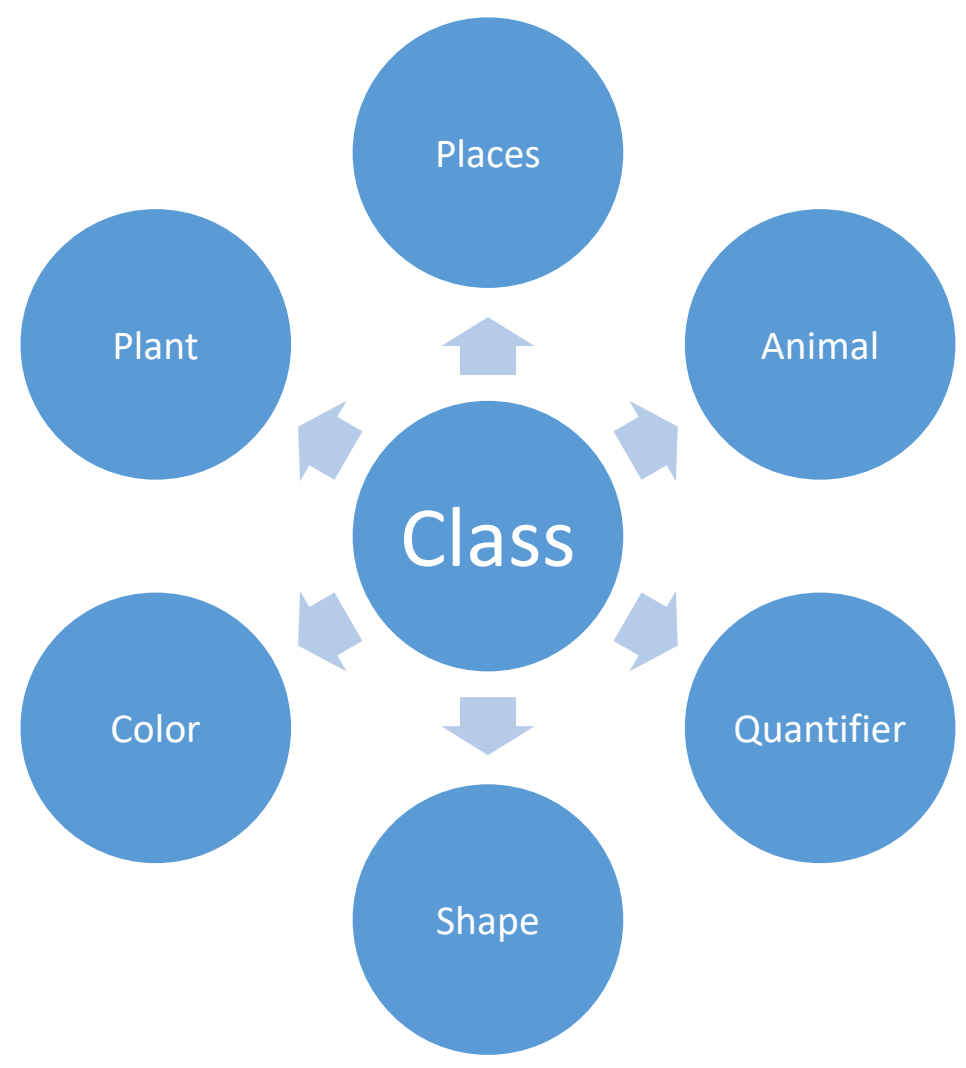

Figure 1: Different categories of medical text translation

There are four main manifestations of this type of medical English: (1) English ordinal numbers, such as first cranial nerve, second cranial nerve ... twelfth cranial nerve is the naming of twelve cranial nerves or the naming and matching of teeth The naming of the viewport; (2) Arabic numerals, such as numbering the chromosomes; (3) Roman numerals, such as type I and type II of 
diabetes; (4) the order by English letters or Latin, Greek letters, for example in Chinese In the case of different types of hepatitis, the order of A, B, C, D and E is expressed. In English, the type of hepatitis is expressed in the order of 26 letters in English, such as Hepatitis A, Hepatitis B, Hepatitis C, Hepatitis D, etc. In addition, some suffixes that represent quantities, such as mono-, bi-, tri-, also appear in medical English ${ }^{[3]}$. Different categories of medical text translation are shown in Figure 1.

\section{Translation strategy of medical English text}

The translation standards of "Faith, Da and Ya" proposed by Yan Fu also have strong practicality and guiding significance in medical translation practice: Medical translation should be "letter", that is, accurately and completely express the original meaning of the text. Medical information has its own unique characteristics compared with other information. If you arbitrarily distorted or changed the meaning of the original text, It will produce serious results. Therefore, for translators, when translating such texts, it is necessary to accurately and clearly express the information contained in the original text. It cannot be ambiguous or ambiguous and it is not allowed to change or distort the original text at will. information. For medical translation, the translated text should be fluent and natural and faithfully express all the information contained in the original text. In the process of translation, not only should the translation and the original text be consistent in form, but also the nature of the translation and the original format cannot be copied, resulting in meaning and ambiguity. Medical translation should also reflect the standard of "elegance", that is to say, the style and style should be consistent with the original text and it should be rigorous, professional and standardized. It must meet the requirements of medical professionalism and different styles according to the style, with reference to its corresponding structure and stylistic specifications for translation. Therefore, in the text translation strategy of medical English, the best translation method should be adopted for the characteristics of different texts in order to achieve the effect of "letter, dad and elegance" on medical English text translation ${ }^{[4]}$. The main points of medical text translation are shown in Figure 2.

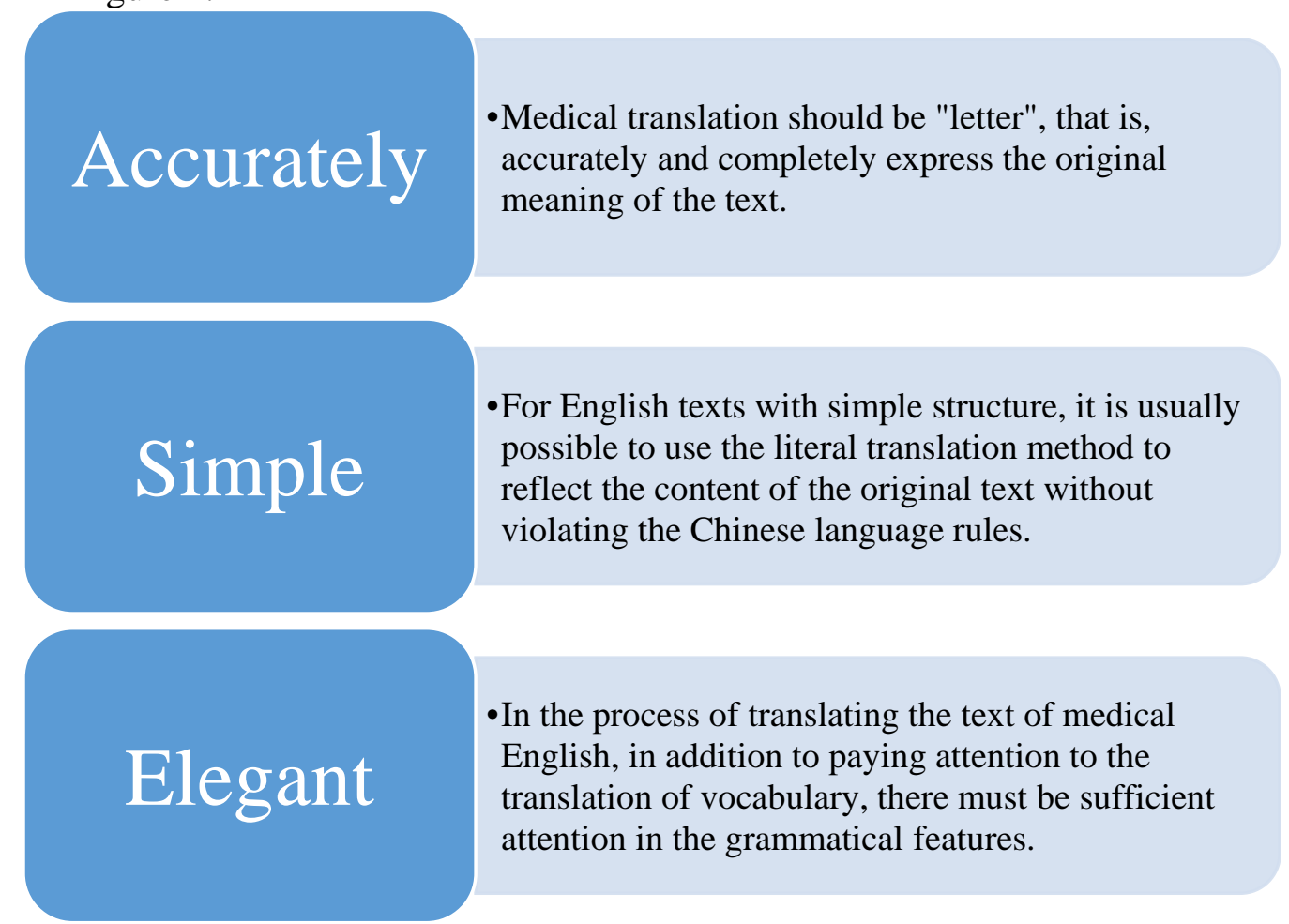

Figure 2 Main points of medical text translation 
For English texts with simple structure, it is usually possible to use the literal translation method to reflect the content of the original text without violating the Chinese language rules, such as rose spot rose rash, canine madness rabies, red blood cell, red blood cell, round worm Etc. But it should be noted that this method is very prone to formalistic death translation, avoiding the situation similar to the translation of banana sign into "banana disease". For the English text of the names of names, the transliteration is usually used to preserve the meaning of the original text more completely, such as Cushing's syn-drome Cushing syndrome, Curie therapy Curie therapy, Radiation therapy, Argentine hemorrhagic fever virus Wait. For English texts that are difficult to express accurately by translating words, usually use the method of free translation to avoid misunderstanding or ambiguity, such as penicillin transliteration "Pencillin", free translation "penicillin"; vitamin transliteration as "vitamin", meaning translated as " Vitamins." For English texts containing letters that express their direct characteristics, pictographic translation is usually used to preserve their characteristics, for example, retaining their morphological features: the capital letter "T" is translated into the Chinese character "D", such as the T-bandage T-belt; "I" is translated as "work", such as I-shaped I-shaped; "Z" is translated as "B", such as Z-iron B-iron; U-shaped plaster cast is translated as "U-shaped plaster support". Retain its style characteristics: spiral fracture spiral fracture; coronary artery coronary artery; splay-foot splayed foot (splay means "open"); spica bondage herringbone (spica means "spike"); pica -roon cross knife (picaroon means "pirate") and so on ${ }^{[5]}$.

In the process of translating the text of medical English, in addition to paying attention to the translation of vocabulary, there must be sufficient attention in the grammatical features and semantic features of medical English. The grammatical feature of medical English texts is that they differ from the translation habits of ordinary English in terms of tense. First, in special circumstances, the passive voice is often used; secondly, in terms of time, in order to prevent misunderstandings, medical accidents and other issues, rarely use the past tense, generally in the present tense; finally, often For the needs of medical argumentation, such as advice and requirements, these are virtual tone, the role of which is to remind patients and medical practitioners. At the semantic level, due to the rigor of medicine, the connection between sentences and sentences is also very strict and coherent. The structure of the article is compact and the use of arranging sentences makes the tone more serious and strong and enhances its persuasiveness.

\section{Conclusion}

There is a fundamental difference between the medical English text and the general English text. It means that it contains both the vocabulary and grammar in the general English text, as well as its own professional terminology, usage and stylistic features. Therefore, the translator It is necessary to accurately grasp the relevant professional knowledge of the medical profession. On the basis of mastering the solid English vocabulary, grammar and semantics, the translator also needs to understand and master the formation and application of medical terminology as much as possible and rationally carry out the medical English text by adopting appropriate translation strategies. translation.

\section{References}

[1] Jafar Asgari Arani. Medical English M-Learning: Positioning a New Paradigm in E-Education[J]. International Journal of Interactive Mobile Technologies,2012,6(1).

[2] Dongsheng Wang, Lijie Wang. Internet-assisted medical English teaching[P]. IT in Medicine and Education (ITME), 2011 International Symposium on,2011.

[3] Yuan Kong. Investigation and Analysis of the Current Situation of Extracurricular English Learning for Contemporary Medical Majors[A]. Shandong University(Weihai).Proceedings of 3rd International Conference on 
Politics, Economics and Law(ICPEL 2018)(Advances in Social Science, Education and Humanities Research, VOL.246)[C].Shandong University (Weihai):2018:5.

[4] Wenjuan Ke. An Empirical Study on Multimodal Teaching Model for Medical English[A]. Singapore Management and Sports Science Institute, Singapore, Hong Kong Education Society, Hong Kong. Proceedings of 2017 5th SSR International Conference on Cultural Studies, Educational Research and Social Sciences(CES 2017)(Advances in Social and Behavioral Sciences,VOL.22)[C].Singapore Management and Sports Science Institute,Singapore,Hong Kong Education Society, Hong Kong:2017:5.

[5] Yuan Kong. Study and Analysis of the Current Situation of Students' Oral English in Medical Colleges and its Countermeasures[A]. Hainan University, Sanya University, Xiamen University Tan Kah Kee College.Proceedings of 4th International Conference on Social Science and Higher Education(ICSSHE 2018)(Advances in Social Science, Education and Humanities Research,VOL.181)[C].Hainan University, Sanya University, Xiamen University Tan Kah Kee College:2018:5. 